\title{
Reflexiones sobre ocupación, identidades culturales y transformación social ${ }^{1}$
}

\author{
Reflections on occupation, cultural identities \\ and social transformation
}

\author{
Nick Pollard ${ }^{2}$ \\ TRADUCCIÓN: \\ Pamela Talero Cabrejo ${ }^{3}$ \\ Clara Duarte Cuervo ${ }^{4}$
}

Pollard, N. (2017). Reflexiones sobre ocupación, identidades culturales y transformación social . (P. Talero \& C. Duarte, Trad.). Revista Ocupación Humana, 17 (1), 55-72.

\section{RESUMEN}

Cuando fui invitado con tanta amabilidad al XVI Congreso Colombiano de Terapia Ocupacional el año pasado en Medellín, una de las impresiones más fuertes de mi corta visita fue el contenido de folclor cultural, a través del uso de la música y la danza, en muchas de las presentaciones. Esto hizo que fuera una experiencia muy diferente a la de otros congresos a los que he asistido. El significado de las culturas parecía ser una parte integral de la práctica profesional, por ejemplo con comunidades indígenas y con poblaciones en áreas rurales, así como del posicionamiento de la Terapia Ocupacional dentro de la historia colombiana reciente. Lo que resultó muy distinto para mí como británico fue que las manifestaciones de música y danza eran algo que todo el mundo parecía conocer y en lo cual todos podían participar. La fortaleza de este aspecto cultural compartido, el cual refleja algunos de los aspectos de la variedad de tradiciones en Colombia, fue cautivador. Esto me llevó a reflexionar sobre el enfoque comunitario de la ocupación humana con propósito, entendido como el hacer colectivo que constituye la cultura. Este artículo de reflexión discute algunos aspectos de la ocupación y la cultura como producto del hacer colectivo para el propósito humano de la comunidad. Se consideran la ocupación y la cultura en oposición a los antecedentes de uso de la ocupación para la salud, y como fundamento de las prácticas socialmente transformadoras. Se esbozan algunos aspectos de la cultura popular de Colombia y el Reino

\footnotetext{
${ }^{1}$ Este artículo se deriva de la video-conferencia preparada por el autor para la jornada "Terapia Ocupacional, cincuenta años transformando conflictos en paz" realizada por el Colegio Colombiano de Terapia Ocupacional el 4 de noviembre de 2016, en el marco de la celebración del día mundial y el día nacional del terapeuta ocupacional.

${ }^{2}$ Terapeuta Ocupacional. Máster en Psiquiatría, Filosofía y Sociedad. Magíster en Terapia Ocupacional. Doctor en Terapia Ocupacional. Profesor Titular Facultad de Salud y Bienestar, Sheffield Hallam University. Sheffield, Reino Unido.

${ }^{3}$ Comité Editorial Revista Ocupación Humana.

${ }^{4}$ Editora Revista Ocupación Humana.
} 
Unido, y algunas razones por las cuales los profesionales deben ser cuidadosos en respetar su integridad, así como su capacidad para la innovación, la adaptación y el cambio como cultura viva.

\title{
PALABRAS CLAVE
}

Ocupación, cultura, música folklórica, narrativas personales

\begin{abstract}
When I was so kindly invited to the XVI CCTO conference this past year in Medellin, one of the lasting impressions of that short visit was the folk cultural content, using music and dance, of many of the presentations. This made it a very different experience to other occupational therapy conferences I have attended. The significance of cultures seemed integral to practice, for example with indigenous people and with people living in rural areas, as well as to the positioning of occupational therapy in its historic place within recent Colombian history. What was very different to me as a British person was that these performances involved something that everyone seemed to know and to be able to participate in. The strength of this shared aspect of culture, which may reflect some aspects of the rich variety of traditions in Colombia, was impressive. It led me to reflect on the community focus of human purposeful occupation as the 'collective doing' that constitutes culture. This reflective paper will discuss some aspects of occupation and culture as the product of collective doing for the community focus of human purpose. It will consider occupation and culture against the background of the use of occupation for health, and as a basis for socially transformative practices. It will draw on some aspects of Colombian and UK folk cultures and some of the reasons why practitioners might be careful to respect the integrity of these assets, as well as their capacity for innovation, adaptation and change as living culture.
\end{abstract}

\section{KEY WORDS}

Occupation, culture, folk music, personal narratives

\section{Introducción}

Bonder, Martin y Miracle (2004) ilustran la importancia de entender la naturaleza de la cultura como algo aprendido, que se desarrolla con el paso del tiempo, posibilita la evaluación, se soporta y se transmite a través de prácticas basadas en la ocupación. Las intervenciones en salud socialmente transformadoras pueden estar basadas en prácticas culturales populares, como la canción y la danza folclórica, así como en prácticas de escritura vernácula. Consideraré tales prácticas en relación con la idea de movimientos sociales (puede argumentarse que este sería el caso de algunas interpretaciones de la cultura popular y las prácticas de escritura y publicación comunitaria de la clase obrera) y las implicaciones que esto puede tener para una práctica socialmente transformadora. La música es una forma cultural comunmente asociada a la narrativa, tanto en su estructura como en el contenido lírico 
de las canciones. Es, entre otras cosas, un medio a través del cual es posible acceder a la memoria, a historias y experiencias con significados personales o colectivos que, por sus contenidos y contextos, pueden eventualmente posibilitar o limitar y excluir (García, 2014). García (2014) señala que buena parte de la exploración de la música como intervención (y de las artes en general) se ha hecho desde una perspectiva jerárquica, y que, por lo tanto, es necesario evaluar estos procesos y entenderlos desde una perspectiva popular. Concluiré explorando las bibliotecas humanas como una forma de práctica basada en la ocupación para trabajar con narrativas a través del diálogo.

Hammel (2009) argumenta que la intervención de Terapia Ocupacional debe respetar el contexto cultural; no obstante, la profesión asume ciertas posiciones respecto a la actividad significativa que no están respaldadas en investigaciones culturales. Por ejemplo, Hong, Heathcote y Hibberd (2011) describen muchas actividades culturalmente aceptadas para trabajar con adultos mayores, pero a pesar de que establecen planes teniendo en cuenta las consideraciones clínicas, prácticamente no se discute la profundidad cultural identificada por Bonder, Martin y Miracle (2004), que podría ser necesaria para suscitar en las personas narrativas que sirvan como base para las artes o la reminiscencia. Sin embargo, desde la perspectiva del desarrollo de actividades de escritura, por ejemplo, Goldblatt (2007), Mathieu, Parks y Rousculp (2012), y Williams (1996) describen la importancia de las prácticas culturales con diferentes comunidades y exponen cómo se pue- de trabajar con la gente para que logre apropiarse de sus creaciones.

Otras ideas equivocadas pueden perjudicar el uso de medios culturales como intervención. García (2014) advierte acerca de la aplicación ingenua de los géneros musicales en los proyectos de reconciliación en Colombia; señala la asociación simplista de la música con el sanar, cuando de hecho puede implicar contenidos y asociaciones complejas, especialmente si se ha utilizado con objetivos políticos o cuando expresa la perspectiva de las víctimas. Esto se evidencia en el poderoso trabajo de las víctimas del conflicto armado en Colombia que se refleja en la videoinstalación Bocas de Ceniza, del maestro Juan Manuel Echavarría (2004), en la que diferentes personas cantan a la cámara. Sus alocuciones están llenas de dolor y sufrimiento. Estas canciones, por toda la verdad que cargan, pueden ser un material con el cual resulte muy difícil trabajar; no pueden ser adoptadas sin respeto hacia las personas que las cantan y sin un poco de consideración hacia las experiencias de quienes puedan escucharlas, especialmente si son los autores de tales atropellos. Como persona que no tiene experiencia en conflictos, puedo escuchar estas canciones y relacionarlas con canciones del folclor británico que conozco y que hablan de desastres, algunas de las cuales pueden contener experiencias de quienes fueron testigos de los mismos; con las experiencias de personas que he conocido, que sobrevivieron a los bombardeos de la Segunda Guerra Mundial o a episodios de los conflictos de Irlanda del Norte (por ejemplo, Sitzia \& Thickett, 2002). Los profesionales no pueden predecir 
cómo responderá la gente al contenido, o lo que puede causar que una persona reflexione sobre algo en lo que estuvo involucrado y reaccione. Los terapeutas ocupacionales a menudo trabajan con personas que tienen historias difíciles que no se pueden reparar. Los usuarios también pueden tener condiciones a largo plazo para las que probablemente no haya solución, pero con las cuales pueden vivir bien (Pemberton, 2014). Así pues, estas intervenciones deben tener la capacidad de mantener a las personas involucradas, de transmitir profundidad y significado, de ofrecer aspectos de apropiación de la participación.

\section{Cultura y comunidad}

La historia del folclor y la cultura vernácula en el Reino Unido es diferente a la de Colombia u otros países en muchos aspectos. A pesar de las relativas diferencias socioeconómicas, son importantes las relaciones históricas entre los grupos culturales del Reino Unido, particularmente la cultura inglesa y el legado del imperialismo han sido fuente de tensiones políticas. Estas relaciones históricas son complejas, pues contienen elementos del folclor que hacen parte de tradiciones oscuras, en ocasiones reinventadas, los cuales se han utilizado para ejemplificar perspectivas nacionalistas (Spracklen \& Henderson, 2013; Palmer Heathman, 2016), pero que también pueden interpretarse de manera progresista (Bose, 2004; Rappaport, 2004). Algunas comunidades en Colombia han desarrollado expresiones musicales de una manera que pretende dar forma y expresar su identidad en resistencia al conflicto, a través de medios narrativos como el rap o el vaIlenato (García, 2014).

Es importante hacer primero una conexión entre cultura y comunidad. La discusión de Hield (2010) sobre la "comunidad popular" en Inglaterra describe las actividades llevadas a cabo en una comunidad con base en sus intereses comunes, no por su ubicación geográfica. La autora retoma la sugerencia de Bauman (2001) respecto a que "comunidad", incluso en el presente, se refiere a una comunidad imaginada de un pasado indefinido. Hield (2010) argumenta que la comunidad de la música popular se delimita "a través de la práctica de cantar un compendio de canciones alusivas en las que [los cantantes] han puesto significado" (p.52). Es en ese repertorio de música popular en el que esas personas parecen basar su identidad y su sentido de pertenencia a una comunidad. Es con una preferencia con lo que se identifican, en lugar de que la música popular sea producto de su identidad. A menudo son cantantes del común que aportan los escasos minutos de su canción a una noche de recital, o tal vez los organizadores de una "sesión" en un bar con una pequeña audiencia (Hield, 2010). En ocasiones he participado en dichas reuniones, los recitales de los grupos editoriales comunitarios y los talleres de escritura en los cuales he estado tienen un sentido similar. Tales alocuciones, ya sean de material original o de canciones muy conocidas, tienen una intimidad particular. A menudo no hay tecnología involucrada, no se necesitan micrófonos y, como eventos regulares en el calendario de recitales, pasan a ser parte de una comunidad de narrativas. 


\section{Intimidad e intercambio}

La capacidad de hacer presentaciones personales, la ubicación de la canción popular o de cualquier otro medio dentro de una narrativa más amplia (incluso si es parcialmente imaginado e idealizado, como exploraremos en un momento), es una parte clave de la cultura. Este intercambio personal de la narrativa es un aspecto importante de la conexión humana, de la construcción de relaciones, volveré a esto más adelante en el artículo. En cada una de estas comunidades de intercambio, ya sea a través de una canción, un poema o una historia, la gente puede tener la sensación de navegar a través de los contenidos de una biblioteca humana. La biblioteca humana es en sí misma un enfoque para la generación de comunidad y la negociación de las diferencias de una manera que podría abordar algunas de las preocupaciones señaladas por García (2014), pero en primer lugar vale la pena explorar cómo las narrativas que pueden hacer parte de tales intercambios están vinculadas a algunos de los elementos tradicionales de la cultura.

Todos estos procesos, el evento de música popular, un taller de escritura, un recital de una editorial comunitaria y las bibliotecas humanas, sugieren una intimidad democrática. Esto es importante para la comunidad, incluso si en realidad estas actividades dependen de algunas personas que se comprometan con su organización y requieran aplicar reglas complejas para ser sostenibles (Hield 2010). Por el contrario, aquellas áreas de la cultura que se materializan en tecnologías y organizaciones comerciales son discutidas por Burgess (2006), pues en lugar de llegar a ser más democráticas, Ilegan a ser demóticas. Sin embargo, aunque muchas expresiones culturales de baja tecnología logran esquivar algunos de los procesos del mercado de consumo, pocas áreas de expresión cultural son completamente independientes de las tendencias dominantes en tecnología, difusión o distribución. El control de los procesos de difusión, edición y aspectos de la alocución son manipulados por los propietarios de los medios de comunicación a través de los cuales se distribuyen; se invita a quienes participan en juegos en línea a desarrollar contenidos, pero dentro de las líneas determinadas por los dueños de los medios. Burgess (2006) presenta la narrativa digital como un ejemplo de las formas en las cuales los individuos pueden producir sus propias narrativas y formas de expresión. Señala que las historias digitales contienen elementos de intimidad a pesar de que emplean temas comunes y de que son específicamente actos individuales de comunicación; pero incluso estas están controladas y limitadas a una audiencia en su distribución, ya que se accede a ellas a través de sitios de narrativa digital. Del mismo modo, la música popular del Reino Unido se ha convertido en interés particular de una comunidad de personas. Estas comunidades son inclusivas, pero operan diversos clubes o "sesiones" que tienen normas de desempeño, de comportamiento e incluso de contenido, aunque puede haber una variedad de formas en las que se puede expresar un significado personal (Hield, 2010). El estudio de Hield sugiere que la localización de algunos de estos públicos puede ser un aspecto que determine la pertenencia a una comunidad de música popular, su regularidad y la sensación común de participación, 
algo que sus miembros regulares valoran como un componente esencial de su vida cotidiana: así operan algunas reglas para preservar su comunidad.

\section{Aplicación y conciencia}

La música popular y la danza se han empleado en la práctica de Terapia Ocupacional (por ejemplo, Connor, 2000; Heathcote \& Hong, 2009), y la participación en la música tradicional ha sido un tema de investigación para los científicos ocupacionales (Adrian, 2013). La literatura anglófona es bastante escasa, pero se remonta a la década de 1950 (Wittkower \& La Tendresse, 1955). Fuentes bibliográficas en español indican alguna aplicación de estas formas, por ejemplo, la enseñanza del flamenco (De Las Heras, 2009) y la investigación etnográfica de la música como componente social y ocupacional importante en la vida de las comunidades indígenas (Arango Peláez, Martin Nieto, \& Rincón González, 2013). Sin embargo, como sugiere Hammell (2009), ha habido poco interés en la aplicación de la música y las artes en Terapia Ocupacional desde el contenido cultural de estos medios, concentrando la atención en los aspectos clínicos de la intervención. Para que estos enfoques sean útiles en la creación de cambios sostenibles es importante entenderlos en su contexto, prestar atención al significado que la música popular y la cultura puede tener para los grupos y las comunidades, y respetar el conocimiento y la tradición que conllevan. Aunque los medios culturales se utilicen como parte de una intervención social o en el trabajo clínico, la preocupación por su autenticidad se aplica en cualquier caso, con quien sea que uno trabaje, ya sea con una población indígena o con residentes en un hogar de ancianos.

Por lo tanto, Ramírez \& Schliebener (2009) han argumentado la necesidad de articular una conciencia ocupacional situada en las realidades específicas de la vida diaria que se reflejan en el contexto cultural latinoamericano. Dehays, Hitchin y Vidal (2012) han argumentado esta necesidad en relación con la práctica centrada en la justicia ocupacional, por ejemplo, en la satisfacción de las necesidades de las madres jóvenes con dificultades de aprendizaje y en la facilitación de la inclusión social de ellas y de sus hijos; Castro (2012), en relación con la construcción de historias de vida con los usuarios de servicios psiquiátricos, y Muñoz (2013), en el bienestar al trabajar con adultos mayores. Una característica de este tipo de estudios es la identificación y el establecimiento de conexiones con comunidades con necesidades complejas que surgen de factores histórico-sociales, económicos y geográficos. Por ejemplo, Zerda (2004) ha señalado que muchas personas mayores en las comunidades de América Latina están alejadas o no pueden acceder a servicios como la Terapia Ocupacional. Los medios culturales son una herramienta importante para que las personas permanezcan involucradas y para superar algunos de los obstáculos que, en otras intervenciones clínicas, surgen de las necesidades y condiciones frente a las cuales se espera que los terapeutas ocupacionales encuentren soluciones.

El reconocimiento de estos problemas más amplios no ha sido parte de la formación de terapeutas ocupacionales. Sin embargo, otros grupos pro- 
fesionales como el Trabajo Social están empezando a explorar lentes como la teoría de la actividad histórico-cultural (CHAT, por sus iniciales en inglés,) para articular asuntos de la práctica con una perspectiva social compleja (Foot, 2001, 2014). La CHAT considera que los seres humanos actúan colectivamente, cooperando en un "sistema de actividad" (Foot, 2014), un término que hace referencia a un proceso completo y complejo, no meramente a los comportamientos que lo componen. Así pues, ya que la gente se comunica a través del hacer, desarrolla herramientas para el aprendizaje y la comunicación, y tiene un enfoque comunitario para todo lo que hace, esto implica que hay muchos elementos que componen la actividad. Foot $(2001,2014)$ describe cómo la CHAT tiene en cuenta las formas en que las experiencias son sentidas y realizadas por la gente. La mediación de la experiencia se produce en las comunidades a través de herramientas como el lenguaje, y finalmente, las formas de expresión cultural. La CHAT ofrece una manera sistemática y crítica de entender la relación entre la cultura y una práctica como la Terapia Ocupacional, y con el tipo de prácticas que se desarrollan en comunidades con intereses específicos, como aquellas que se involucran en la música popular en el Reino Unido. Para los terapeutas ocupacionales, la aplicación simplista de una actividad como la escritura (PoIlard, 2004) para lograr un resultado terapéutico puede ignorar otros elementos importantes en la mediación de las experiencias. La CHAT es un vehículo para la exploración de las contradicciones y las tensiones dialógicas que puedan surgir al usar una forma cultural como la música o la danza en un sistema de actividad clínica. En particular, ofrece la posibilidad (ya que un resultado probable de su uso puede ser la producción de cambios) de considerar futuros desarrollos, desde una perspectiva de evolución en el tiempo. Estas consideraciones son importantes si los profesionales van a examinar cómo un objeto como la música puede emplearse como actividad y cuáles pueden ser sus roles al implementarla (Foot, 2014).

\section{Proceso orgánico}

Aunque estas consideraciones son importantes, quiero discutir específicamente los elementos orgánicos del proceso popular en relación con la ocupación significativa para las personas. Cuando alguien adapta una canción popular con su propia versión, está haciendo algo que es parte natural de dicho proceso; por ejemplo, la adaptación por parte del cantante del contenido o de la interpretación para ciertas audiencias o propósitos. Como describe Hield (2010), una canción puede llegar a ser reconocida localmente como propiedad de ciertos cantantes a través de un proceso en el que ellos la apropian luego de ensayarla y presentarla en espacios comunitarios. Eyerman (2002) explora cómo algunas formas tradicionales se fueron adaptando durante varias etapas y generaciones, para finalmente ser usadas en el movimiento de derechos civiles afroamericano en los Estados Unidos de América. El hecho de partir de una base de conocimiento tradicional que históricamente ha pertenecido al pueblo es una fuente de fortaleza. Esto hace posible que se dé un proceso en el cual ocurren interacciones de "intelectuales orgánicos" (Rappaport, 2004, p.113) con otros grupos de "nuevos intelectuales", al servicio de 
lo que Gramsci denominó "la sociedad política" o la "sociedad civil" (1971, p.12). Los nuevos intelectuales Ilevaron a cabo las funciones que requería la sociedad civil, pero al mismo tiempo se permitieron tomar una distancia crítica debido a su posición mediadora. Quienes prestan el servicio de Terapia Ocupacional, por ejemplo, continúan las funciones de estructuras hegemónicas, como los sistemas de salud y de atención social, pero también responden a las experiencias de las personas que reciben dicho servicio. Quizás de manera similar, Peloquin (2010) apunta al ethos de la Terapia Ocupacional, un conjunto de creencias que guían la práctica a partir de sus necesidades, pero también un sentido de compromiso con el mundo real.

Una limitación del pensamiento de Gramsci (1971) es que asume que la gente que trabaja con los intelectuales no es capaz, por sí misma, de objetivar y críticar sus experiencias. No sería entonces aconsejable mantener esta perspectiva al trabajar con un grupo, como lo indican Mathieu, Parques y Rousculp (2012) respecto a la publicación comunitaria. Goldblatt (2007) describe cómo incurrir en ese error puede generar reacciones difíciles en miembros de la comunidad, que demandarían un arduo trabajo para restablecer la confianza. Las perspectivas indígenas del mundo real requieren una comprensión comprometida, no solo en términos del lenguaje, sino desde una visión trasversal de la vida en su totalidad (Rappaport, 2004). Las formas culturales como la música son parte importante de los procesos de transformación social, la música popular ha sido, por mucho tiempo, un vehículo para articular perspectivas críticas, alternativas, o como en el ejemplo de "Spencer the Rover" que discutiré más adelante, marginales. Sin embargo, este tipo de trabajo rápidamente revela una serie de aspectos en los cuales la cultura vernácula se encuentra con los posibles agentes de cambio y sus intentos de transformación; la organización, desarrollo y sostenibilidad de tales aspectos requiere una negociación con los propietarios de algunos de esos espacios. Para aqueIlas personas cuyas tradiciones culturales serán vehículos de una nueva tradición crítica, las habilidades formales de comunicación, presentación adecuada y contabilidad financiera pueden resultar menos conocidas. Las relaciones de poder que resultan de que algunas personas tengan esas habilidades y otras no, pueden ser una fuente de sospecha y hostilidad, especialmente cuando es necesario emplear personas para organizar el trabajo en lugar de depender de voluntarios. Tal como lo evidenció Woodin (2007) al trabajar con la escritura y la publicación comunitaria de la clase obrera: puede haber sospechas de que las personas de clase media se apropien del trabajo creativo de los miembros del grupo de la clase obrera y lo exploten para su propio beneficio, o como Bose (2004) argumenta, que los intelectuales pongan sus argumentos en las voces de los demás, planteando interrogantes acerca de la legitimidad y la suplantación (Eyerman, 2002).

\section{Tradición e invención}

La cultura inglesa está imbuida en muchos años de tradición. Sin embargo, lo que a menudo se transmite como emblemático del país es la asociación con la pompa y la ceremonia de la élite, gran parte de la cual, en realidad, 
fue inventada para instaurar una identidad británica en los siglos XIX y XX (Cannadine, 1983). Gran parte de la cultura tradicional de la gente del común no es estática ni fija. Las colecciones de música popular, como la editada por Hall (1998), indican una gama considerable de formas y estilos, pero siglos de mestizaje han ensombrecido buena parte de sus orígenes. Aunque no se compara con la enorme variedad de formas y tradiciones indígenas de Colombia (Gil, 2011), Hield (2010) y Cressy (2004) sugieren un patrón de reinvención continua, transmitida principalmente por vía oral y a través de la interpretación. Las tradiciones de la música popular inglesa, el baile Morris y el canto de villancicos se mantienen en algunas comunidades, pero la relación entre el canto y la cultura cotidiana más amplia sugiere que el término "tradicional" hace referencia a un pasado idealizado (Hield, 2010).

La música popular tiene sus raíces en la experiencia de las comunidades a las que pertenece y en la expresión de narrativas ocupacionales. Uno de los temas más comunes en esas expresiones se refiere al cambio. La música popular a menudo registra recorridos o experiencias más personales de transición, este es un elemento común en la naturaleza experiencial y autobiográfica de la escritura de la clase obrera (Ragon, 1986; Vincent, 1981). Ragon, al escribir acerca del surgimiento de la literatura proletaria al final del siglo XVIII y en el XIX, explica como esta fue estimulada directamente por las experiencias de industrialización y el aumento de la confianza entre algunos trabajadores, pero también por vivencias de pobreza y privación de derechos. Hay elementos en los que muchas canciones populares pueden tener afinidad - aunque la autoría de muchas de ellas sea incierta. El tema de volver a casa es recurrente en las canciones y en las narrativas populares, y evoca la idea de la vida como un viaje.

Spencer The Rover "who had traveIled Great Britain and most parts of Wales $s^{5 \prime}$ (quien ha viajado por Gran Bretaña y la mayor parte de Gales)" (Pollard, 1969, p. 67) es una balada inglesa ampliamente difundida, una canción que cuenta una historia (Roud, 2011). La historia de transición y cambio de Spencer puede tener su origen en los primeros años de 1800, quizás esté asociada con su salida del ejército o con la pérdida del trabajo en una de las nuevas fábricas de Yorkshire. Estas circunstancias pueden haber generado su aislamiento, su angustia mental, la resolución y la reconciliación que se describen como: "been so reduced which caused great confusion/ And that was the reason he went on the roam" (lo habían diezmado de tal manera que le causaban gran confusión/Y por esa razón se fue a vagar), lejos de su esposa y sus hijos. La manera como concluye la canción, cuando él llega a casa y a pesar de todo es aceptado de nuevo por su familia, es casi bíblica, como el cuento del hijo pródigo, al cual se asemeja en algunos aspectos, en tanto a Spencer su familia le perdona haber perdido su riqueza, demostrando que las relaciones tienen más importancia que lo material. Es una de mis favoritas - mi padre solía cantar fragmentos,

${ }^{5}$ Nota de las traductoras: se conserva la canción en su idioma original, la traducción al español aparece entre paréntesis. 
aunque la aprendió de un disco y la incluyó en una compilación de canciones populares británicas para las escuelas que publicó en la década de 1960, parece un testimonio real. Spencer the Rover es de una época más rural, a pesar de la creciente industrialización, un periodo en el cual resultaba más factible, incluso aceptable, "ir sin rumbo" y vivir como un vagabundo buscando trabajo donde lo encontraras; era el inicio de la época de la industrialización, cuando muchas personas se estaban moviendo hacia las ciudades en busca de trabajo. Eso sería mucho más difícil de hacer hoy en día; al Spencer de hoy se le pediría que sentara cabeza.

Spencer the Rover ofrece una transformación idílica y romántica, del problemático indigente al hombre de familia aceptado y contento. En cierto modo, resume una de las creencias que están detrás de muchas intervenciones culturales: que, de alguna manera, si se puede descubrir una base de expresión comunitaria, esto dará lugar a una comunidad utópica y a la felicidad, como en tiempos pasados. La evidencia histórica es mucho más problemática de lo que indica Spencer. Otros, en el canon folclórico más amplio (por ejemplo, Hall, 1998; Roud, 2011), describen algunos de los peligros que experimentan las personas que trabajan: el transporte, la pobreza, una amplia gama de delitos, malas y duras condiciones de trabajo, el reclutamiento forzado, así como la exposición a desastres y otros extremos que no están muy lejos de las voces exploradas por García (2014) o Echavarría (2004).

Los elementos conservadores de la música popular inglesa pueden hacer de ella un aspecto controversial de la cultura británica, y la mantienen como un medio difícil de emplear en la super-diversidad (Vertovec, 2007) de la sociedad británica contemporánea. El interés en las culturas populares ha resurgido en varias oportunidades desde finales del siglo XIX. En algunos de esos momentos se ha tendido a interpretar la música popular en términos útiles a los intereses contemporáneos o particulares de quienes buscan revivirla (Brocken, 2003). La música popular inglesa, por ejemplo, ha sido adoptada por ciertos renovadores como emblemática de una expresión nacionalista. Coleccionistas de los años 1920 y 1930 trataron de recoger formas de canto y danza tradicionales en una perspectiva de extrema derecha de "blancura", de un inglés robusto y rural, en ocasiones acompañada de una asociación esotérica con "magia blanca" (Spracklen \& Henderson, 2013; Palmer Heathman, 2016). Esto, y una representación generalizada de los entusiastas de la música popular como opositores obsesivos y marginales al mundo moderno siguen siendo obstáculos para que la expresión popular tradicional inglesa sea aceptada. La necesidad de hacer hincapié en lo inglés aquí obedece a la existencia de diferentes tradiciones populares británicas, escocesas, gaélicas, galesas e irlandesas, así como inglesas, con variaciones regionales y una gran cantidad de material compartido. Las diferencias entre ritmos, melodías y contenidos son evidentes, pero ha habido una tendencia particular a escuchar, incluso en Inglaterra, más que la canción tradicional inglesa -tal vez por las razones antes descritas-, un folclor irlandés popularizado, a pesar de que a veces tenga letras románticas nacionalistas y sectarias (Smyth, 2004). Este fenómeno pudo haberse propagado por 
cuenta de las sesiones de música que realizaban los artistas irlandeses que emigraron a Londres en la década de 1950 (Kearney, 2007). Esta tradición, por ejemplo, sobrevive en los bares irlandeses de Sheffield, pero se ha extendido por el mundo.

\section{Canciones para la transformación social}

A pesar de estas cuestiones, Palmer Heathman (2016) sostiene que las ideas de transformación social, representadas también en estos movimientos, se basaban en una apelación al pasado que posibilitaría un futuro utópico y comunitario. La autora argumenta que los resurgimientos populares también buscaban generar una vinculación entre las personas inspirada por el amor a la nación, un punto que Kearney (2007) también señala en relación con una tradición, menos formal, de emigrantes irlandeses. El futuro dependía de que las raíces volvieran a la vida, y esas raíces estaban en la tradición, la nacionalidad y la comunidad. De hecho, como lo señalan Arango Peláez, Nieto Martín y Rincón González (2013) en su estudio sobre los muiscas, la música se utiliza para celebrar la unidad entre un pueblo, su entorno y su cosmos. Aunque algunas de estas conexiones pueden resultar extrañas en los usos urbanos de la música popular en el Reino Unido, tales raíces aún pueden celebrarse en los espacios comunes con el fin de crear comunidad (Hield, 2010; Spracklen \& Henderson, 2013; Palmer Heathman, 2016), al igual que en Colombia (Gil, 2011).

Estos espacios comunitarios son, en sí mismos, espacios de considerable cambio e influencia global (Gil, 2011). Dada la diversidad de las tradiciones musicales de Colombia, el énfasis está más en disfrutar y apreciar su variedad y las nuevas posibilidades que presentan, que en ligarlas con la tradición. Esta es sin duda mi experiencia personal con la reproducción del folclore británico en los bares, y tal vez la de muchos artistas, no obstante, puede resultar doloroso para las organizaciones que prefieren la música tradicional a la innovación (Hield, 2010; Spracklen \& Henderson, 2013). El arraigo es esencial, tanto para la identificación de los procesos de cambio como para el uso de formas y estructuras reconocidas como medio. Estas no pueden ser adaptadas de manera genuina sin respetar sus contextos, de lo contrario, no es más que explotación. Para la música popular sigue existiendo una tradición viva, como exploraremos más adelante, pero las tradiciones vivas en la actualidad tienen una relación difícil con las fuerzas comerciales del mercado, las cuales no solo las graban e interpretan, sino que introducen al repertorio material nuevo o imitativo.

\section{Movimientos sociales y prácticas culturales}

Tanto Brocken (2003) como Gil (2011) sugieren que los cambios y las influencias globales han sido un factor clave en el desarrollo de la música popular. El hecho de que algunas canciones, ritmos y formas se popularicen sugiere que no son fijos, sino producto de cambios sociales. En el Reino Unido ha habido una cierta resistencia a la influencia extranjera y comercial, lo que resulta en lo que Hall (1998, p.5) ha Ilamado "moralismo cultural", una 
insistencia alienante en un tradicionalismo fijo (Brocken, 2003). Estas tensiones hacen parte de un proceso en el cual las acciones de cambio están vinculadas a movimientos sociales, especialmente a aquellos que tienen un propósito creativo y cultural en torno a la generación de una identidad Colectiva (Melucci, 1985; Martin, 2002). Naturalmente estas nuevas formulaciones de identidad colectiva son ensamblajes sociales complejos y frágiles a la vez, que requieren que quienes se adhieren a ellos los repitan y definan con el fin de mantenerlos vivos, como en el caso del moralismo cultural que sin duda moldeó mi interés por la música. Mientras en la década de 1960 otros niños crecieron con los Beatles y Motown, yo crecí con la música popular tradicional de The Watersons (ej. 1965) y Shirley Collins (ej. 1967). En las clases de música de la escuela primaria en las que aprendíamos canciones populares, me decepcionaba que se esperara que las cantáramos con una armonía convencional y no con las voces naturales, a veces deliberadamente "naturales", que acostumbraba a escuchar en el tocadiscos de papá.

Este tipo de música todavía se toca ocasionalmente en los bares, junto a melodías más populares que se han adoptado democráticamente en el repertorio popular; lo "popular" desafía cualquier categorización (Hield, 2010). Como Gil (2011) lo argumenta respecto a Colombia y Hield (2010) lo sugiere para Inglaterra, la variedad de tradiciones disponibles en un contexto musical globalizado invita a los jóvenes a experimentar y a las generaciones mayores a ceder ante la disonancia.

La música popular es ecléctica, es ante todo variación. Existen múltiples versiones de canciones, incluyendo algunas que parecen ser mezclas de versos estándar. Las mismas palabras pueden ser compartidas por las letras de diferentes canciones, otras canciones pueden ser interpretadas usando diferentes melodías. Como algunos cuentos populares, ciertas canciones populares han viajado alrededor del mundo. Cualquiera que cante una canción debe ser capaz de mantenerla viva a través de su propia interpretación (Hield 2010), algo que mi padre Ilamaba "el proceso popular" (Pollard, 1969). Este cambio, adaptación y reinvención del contenido en sí mismo como acción social (Eyerman, 2002; Gil, 2011) es, además de la acción de cantar e interpretar, un aspecto cultural importante de la ocupación (Guajardo \& Mondaca, 2016), así como la expresión de la vida cotidiana, de los acontecimientos importantes de la vida y de la experiencia del cambio.

\section{Bibliotecas Humanas}

Este aspecto del descubrimiento de nuevas posibilidades culturales, y la aceptación y el interés por la diversidad han sido importantes para otros movimientos relacionados con la expresión cultural. El movimiento editorial comunitario del Reino Unido tenía que ver con la celebración de los lugares y con el descubrimiento de sus culturas e historias; se llevó a cabo en espacios como salones comunales, bares y librerías (Morley y Worpole, 2009).

Un desarrollo más reciente que surge a partir de los relatos de la gente del común es la Biblioteca Humana. Este enfoque dialógico no ha sido abordado 
en la literatura de Terapia Ocupacional, aunque algunas bibliotecas públicas han desarrollado proyectos en torno a intervenciones comunitarias. Little, Nemutlu, Magic y Molnár (2011), y Little y Abergel (2013) describen la Biblioteca Humana como un medio a través del cual "libros" humanos pueden ser tomados en préstamo por los lectores durante cortos períodos de tiempo. Los libros pueden tener títulos como "drogadicto", "solicitante de asilo" o "guardia de tráfico", pero deben ser simples y transmitir la principal experiencia que estén dispuestos a compartir al interactuar con los lectores. El proceso de "préstamo" es mediado por un "bibliotecario", un "casamentero" que asigna los libros a los lectores, y por los auxiliares de biblioteca, quienes explican su funcionamiento a quienes quieren ser lectores. Los libros se asignan a los lectores, pero ambos pueden hacerse preguntas entre sí. Las personas pueden aprender unas de otras a través de la conversación, con el objetivo de compartir puntos de vista y experiencias personales, tomar conciencia y superar sus prejuicios y estereotipos. Las bibliotecas humanas ofrecen interacción a través del diálogo, más que historias, lo que de alguna manera compensa las preocupaciones expresadas por García (2014) en relación con el contenido narrativo; sin embargo, pueden no ser aplicables a algunas situaciones, por ejemplo, cuando las personas recientemente han experimentado hechos traumáticos. Los eventos de bibliotecas humanas pueden ligarse a otros tales como festivales de rock o congresos juveniles, o asociarse con eventos de la comunidad. Garbutt (2016) describe la organización de bibliotecas humanas en asociación con un incremento del nacionalismo en Australia alrededor del conflicto en Afganistán. No hay temas, los eventos de la biblioteca no se organizan en torno a temas individuales, grupos o ideologías; el enfoque permite que cualquier persona que pueda experimentar prejuicios pueda hacer parte en forma de libro, siempre y cuando esté dispuesta a compartir sus experiencias con un lector. El énfasis está en la inclusión sobre la exclusión, pero requiere de capacidad para compartir, desafiar los prejuicios y ser desafiado; por lo tanto, debe llevarse a cabo en un espacio público que permita el desarrollo de múltiples discusiones.

Goebel (2011) informa que es importante tener una sesión de orientación para las personas que se ofrecen como voluntarias para ser "libros", de modo que puedan gestionar y ensayar las narraciones de las experiencias que van a contar de manera más efectiva. Tal vez sea la primera vez que las están contando, y aunque es posible rechazar ciertas preguntas, la situación podría dar un giro inesperado. Little, Nemutlu, Magic y Molnár (2011) ponen de manifiesto que la metodología de la Biblioteca Humana debe seguirse cuidadosamente, esto es importante para la seguridad de los participantes.

Los eventos de la Biblioteca Humana se han organizado en centros educativos con el fin de ampliar entre los estudiantes sus perspectivas respecto a posibles fuentes de información, y puede ser un precursor de otros procesos de asociación en el desarrollo de proyectos comunitarios. Una ventaja considerable de este enfoque dialógico es que las personas no tienen que saber leer o escribir, ni requieren un excelente dominio del idioma, necesitan tener la voluntad de compartir e intercambiar 
experiencias auténticas. Una de mis colegas está organizando eventos de bibliotecas humanas con los usuarios de salud mental y cuidadores en el pueblo de Sleaford, Lincolnshire. Ella ha descubierto que para tales grupos quizás se requiera adaptar ciertas partes del proceso, por ejemplo, ser un "libro" durante 15 minutos y no durante 30, como se sugiere en las directrices; esto asegura un intercambio suficiente, sin correr el riesgo de quedarse sin tema de conversación. Los "libros" pueden necesitar un descanso entre un préstamo y el otro, los descansos frecuentes incrementan las oportunidades de trabajo en red. Sin embargo, tratar de estimar cuántas personas van a participar en un evento que se divulga de boca en boca y con métodos de difusión de bajo costo, es imposible. Se necesita entonces una flexibilidad planificada, puede ser necesario intercambiar los roles de "libros" y "lectores", de modo que todo el mundo pueda participar de diferentes maneras. Coordinar un evento de este tipo implica comprometerse con un proceso incierto, pero orgánico. Es una aventura, una exploración; el proceso en sí mismo supone un elemento de asunción de riesgos y de confianza. Inclusive, puede llevar a descubrir a los intelectuales orgánicos de la comunidad.

\section{Conclusión}

La cultura puede ser vista como la expresión o el producto del hacer colectivo que resulta del enfoque de la comunidad en el propósito humano. Allí donde las intervenciones ocupacionales se usan para promover la salud, puede cobrar importancia que sean auténticas respecto a las expe- riencias de las personas a las que involucran, especialmente que parezcan reales en el contexto en el que se desarrollan, para que tengan afinidad con las prácticas sociales transformadoras, pero no tanto que resulten restrictivas. Las culturas de Colombia y el Reino Unido son distintas, pero es importante apreciar el contenido y la integridad de estas formas, así como su capacidad de innovación, adaptación y cambio como culturas vivas.

\section{Referencias}

Adrian, A. (2013). An Exploration of Lutheran Music-Making among US Immigrant and Refugee Populations. Journal of Occupational Science, 20 (2), 160-172.

Arango Peláez, J.A., Nieto Martin, J.O. \& Rincón González, F.A. (2013). Transformación ocupacional en hombres y reconocimiento de la memoria indígena muisca "Cabildo de Bosa" (Tesis de pregrado). Bogotá: Universidad Nacional de Colombia.

Bauman, Z. (2001). Community: Seeking Safety in an Insecure World. Oxford: Polity.

Bonder, B. R., Martin, L., \& Miracle, A. W. (2004). Culture emergent in occupation. American Journal of Occupational Therapy, 58, 159-168.

Bose, P.S. (2004), Critics and experts, activists and academics: Intellectuals in the fight for social and ecological justice in the Namada Valley, India. En M. Baud y R. Rutten (eds.) Popular intellectuals and social movements: Framing protest in Asia, Africa, and Latin America (pp.133-157). Cambridge: Cambridge University Press. 
Brocken, M. (2003). The British Folk Revival: 1944-2002. Aldershot: Ashgate.

Burgess, J. (2006). Hearing ordinary voices: Cultural studies, vernacular creativity and digital storytelling. Continuum, 20(2), 201-214.

Cannadine, D. (1983). The context, performance and meaning of ritual: The British monarch and the 'invention of tradition 1820-1977. En E. Hobsbawm (ed.) The invention of tradition (pp.101164). Cambridge: Cambridge University Press.

Castro, L.R. (2012). Construcción de historias de vida: una evaluación narrativa para la intervención de terapia ocupacional en personas con discapacidad psiquiátrica. Revista electrónica de Terapia Ocupacional Galicia, TOG, (16), 11-11.

Collins, S. (1967). The sweet primeroses. London: Topic Records.

Connor, M. (2000). Recreational folk dance: A multicultural exercise component in healthy ageing. Australian Occupational Therapy Journal, 47( 2), 67-76.

Cressy, D. (2004) Bonfires and bells: National memory and the protestant calendar in Elizabethan and Stuart England. Stroud: Sutton.

Dehays, M., Hichins, M. \& Vidal, V. (2012). Análisis del significado de las ocupaciones atribuidas a ser mujer y madre para mujeres con discapacidad intelectual en la ciudad de Punta Arenas. Revista Chilena de Terapia Ocupacional, 12(2). Disponible en: http://200.89.78.45/index.php/RTO/article/viewArticle/25301
De-las-Heras, B. (2009). “Ell@ s" también pueden bailar flamenco. En El largo camino hacia una educación inclusiva: la educación especial y social del siglo XIX a nuestros días: XV Coloquio de Historia de la Educación, Pamplona-Iruñea, 29, 30 de junio y 1 de julio de 2009 (pp. 477-486). Universidad Pública de Navarra.

Echavarría, J. M. (2004). Bocas de Ceniza (Mouths of Ash). https://vimeo. com/31130555 .

Eyerman, R. (2002). Music in Movement: Cultural Politics and Old and New Social Movements. Qualitative Sociology, 25 (3), 443-458.

Foot, K. A. (2001). Cultural-historical activity theory as practice theory: illuminating the development of conflictmonitoring network. Communication Theory, 11(1), 56-83.

Foot, K. A. (2014). Cultural-historical activity theory: Exploring a theory to inform practice and research. Journal of Human Behavior in the Social Environment, 24(3), 329-347.

Garbutt, R. (2016). Everyday peace, human rights, belonging and activism in a 'peaceful' nation. En G. Bee Chen, B. Offord \& R. Garbutt (eds.) Activating Human Rights and Peace: Theories, Practices and Contexts (pp.143-158). Abingdon: Routledge.

García, M. E. P. (2014). Music and reconciliation in Colombia: opportunities and limitations of songs composed by victims. Music and Arts in Action, 4(2), 24-51. 
Gil, G.A.L. (2015). ¿Música vieja, música nueva? Procesos de cambio cultural en la práctica de las cuerdas tradicionales andinas de Colombia, transición al siglo XXI. Artes la Revista,10(17), 140157.

Goebel, N. (2011). Fags, Blacks and Hutterites: Challenging Prejudice and Stereotypes with the augustana human library. Disponible en http://s3.amazonaws.com/ academia.edu.documents/17533195/ fags_blacks.pdf?AWSAccessKeyl$\mathrm{d}=$ AKIAJ56TQJRTWSMTNPEA\&Expires $=1473079421 \&$ Signature $=8 \times A R U$ hKvRDfOT\%2F\%2BJr2Xv2v3CzIY\%$3 \mathrm{D} \&$ response-content-disposition=inline\%3B\%20filename\%3DFags_Blacks_ and_Hutterites_Challenging_P.pdf

Goldblatt, E. (2007). Because we live here: Sponsoring literacy beyond the college curriculum. New Jersey: Hampton Press.

Gramsci, A. (1971). Selections from the prison notebooks. Editado y traducido por Quintin Hoare y Geoffrey Nowell-Smith. London: Lawrence and Wishart.

Guajardo, A. \& Mondaca, M. (2016). Human rights, occupational therapy and the centrality .of social practices. En D. Sakellariou, N. Pollard (eds.) Occupational therapies without borders: Integrating justice with practice (pp.102108). Edinburgh: Elsevier

Hall, R. (1998) Introduction to the series. En R. Hall (ed.) The Voice of the People (pp.3-10). London: Topic Records.

Hammell, K.W., 2009. Sacred texts: A sceptical exploration of the assumptions underpinning theories of occupation. Canadian Journal of Occupational Therapy, 76(1), 6-13.
Heathcote, J. \& Hong, C.S. (2009). Groupwork as a tool to combat loneliness among older people: Initial observations. Groupwork, 19(2), 121-130.

Hield, F. (2010). English folk singing and the construction of community ( $\mathrm{PhD}$ thesis). University of Sheffield, Sheffield, United Kingdom. Disponible en http://etheses.whiterose.ac.uk/1544/4/ Hield,_Fay.pdf

Hong, C.S., Heathcote, J., \& Hibberd, J.M. (2011). Group and individual work with older people. London: Jessica Kingsley.

Kearney, D. (2007). (Re)locating Irish Traditional Music: Urbanising Rural Traditions. Critical Public Geographies (Documento de trabajo UCC Cork Ireland) Disponible en http://s3.amazonaws.com/ academia.edu.documents/30841616/ kearney.pdf?AWSAccessKeyId=AKIAJ 56 TQJRTWSMTNPEA\&Expires $=1474218395 \&$ Signature $=\mathrm{Cl} \% \%$ 2 BKSv\%2B\%2BRiGi7Wra95VOXtn$\mathrm{mWC} \% 3 \mathrm{D} \&$ response-content-disposition=inline $\% 3 \mathrm{~B} \% 20$ filename\%3DRe_ locating_Irish_Traditional_Music_Urb. pdf

Little, N., Nemutlu, G., Magic, J., \& Molnár, B. (2011). Don't judge a book by its cover! The Living Library Organiser's Guide 2011. Budapest: Youth Department of the Council of Europe. Disponible en https://www.coe.int/t/ dg4/eycb/Source/EYCB\%20Living\%20 Library.pdf

Little, N. \& Abergel, R. (2013). Human library. A short guide for Organisers. Copenhagen: The Human Library Organisation/The Outsiders. 
Martin, G. (2002). Conceptualizing Cultural Politics in Subcultural and Social Movement Studies. Social Movement Studies, 1(1), 73-88. DOI: http://dx. doi.org/10.1080/14742830120118909

Mathieu, P., Parks, S., \& Rousculp, T. (eds.). (2012). Circulating communities: The tactics and strategies of community publishing. Lanham, Maryland: Lexington Books.

Melucci, A. (1985). The Symbolic Challenge of Contemporary Movements. Social Research, 52(4) 789-816.

Morley, D. \& Worpole, K. (2009). The republic of letters. $\left(2^{\mathrm{a}}\right)$ edición. Philadelphia/Syracuse New City: Communities Press/Syracuse University Press.

Muñoz, C. (2013). Bienestar subjetivo y actividad social con sentido histórico en adultos mayores. Hacia la promoción de la salud, 18(2), 13-26.

Palmer Heathman, K. (2016). Revival: The Transformative Potential of English Folksong and Dance, 1890-1940 (Tesis no publicada). University of Leicester, United Kingdom. Disponible en https://Ira.le.ac. uk/bitstream/2381/37960/1/2016PALMERHEATHMANKBPhD.pdf

Peloquin, S. (2010). An ethos that transcends borders. En F. Kronenberg, N. PoIlard, D. Sakellariou (eds.) Occupational therapies without borders: Towards an ecology of occupation-based practices (Volume 2) (pp 57-64). Edinburgh: Elsevier Science.

Pemberton, S. (2014). Long-term conditions - condition management or managing to live? British Journal of Occupational Therapy, 77(5), 221
Pollard, M. (1969). Ballads and Broadsides. London: Pergamon.

Pollard, N. (2004). Notes towards an approach for the Therapeutic Use of Creative Writing in Occupational Therapy. En F. Sampson (ed) Creative Writing in Health and Social Care (pp.189200). London: Jessica Kingsley.

Ragon, M. (1986). Histoire de la Litterature Proletariene de Langue Francaise. Paris: Albin Michel.

Ramírez, R. \& Schliebener, M. (2009). Ocupación y literatura, un análisis desde la dialéctica materialista. Revista Chilena de Terapia Ocupacional, (9), 167. Disponible en: http://www.revistaderechopublico.uchile.cl/index.php/RTO/ article/viewArticle/121

Rappaport, J. (2004). Between sovereignty and culture: Who is an indigenous intellectual in Colombia? In M. Baud and R. Rutten (eds.) Popular intellectuals and social movements: Framing protest in Asia, Africa, and Latin America (pp.111-132). Cambridge: Cambridge University Press.

Roud, S. (2011). Good people take warning: Ballads sung by British and Irish traditional singers. London: Topic Records.

Sitzia, L. \& Thickett, A. (2002). Seeking the enemy. London: Working Press.

Smyth, G. (2004). The Isle is full of noise. Irish Studies Review, 12(1), 3-10.

Spracklen, K. \& Henderson, S. (2013). “Oh! What a tangled web we weave": Englishness, communicative leisure, identity work and the cultural web of the English folk morris dance scene. Leisure/Loi- 
sir, $37(3), 233-249$.

Vertovec, S. (2007). Super-diversity and its implications. Ethnic and Racial Studies, 30(6), 1024-1054, DOI: http://dx. doi.org/10.1080/01419870701599465

Vincent, D. (1981). Bread, knowledge and freedom, a study of ninteenth-century working class autobiography. London: Europa Publications.

The Watersons (1965). Frost and Fire: A calendar of ceremonial folk songs. London: Topic Records.

Williams, J. (1996). Across the street, around the world: A handbook for cultural exchange. London: British American Arts Foundation.
Wittkower, E.D. \& La Tendressse, J.D. (1955). Rehabilitation of chronic schizophrenics by a new method of occupational therapy. British Journal of Medical Psychology, 28,(1), 42-47.

Woodin, T. (2007): 'Chuck out the teacher': radical pedagogy in the community. International Journal of Lifelong Education, 26 (1), 89-104. DOI: http://dx. doi.org/10.1080/02601370601151471

Zerda, M. (2004). Vejez y pobreza en Bolivia: La visión de las personas de edad. Disponible en http://hostinweb. es/fiapam/vejez_y_pobreza\%20(Bolivia)\%20Merce_.pdf 